\title{
Suppressive effects of saliva against enamel demineralization caused by acid beverages
}

\author{
Shoji Takahashi ${ }^{1^{*}}$, Shigeru Watanabe ${ }^{1}$, Takashi Ogihara $^{1}$, Koji Watanabe $^{1}$, Kun Xuan $^{2}$, \\ Xiaojing Wang ${ }^{2}$ \\ ${ }^{1}$ Division of Pediatric Dentistry, Department of Human Development \& Fostering, School of Dentistry, Meikai University School of \\ Dentistry, Sakado, Japan; *Corresponding Author: s-takahashi@dent.meikai.ac.jp \\ ${ }^{2}$ Department of Pediatric Dentistry, College of Stomatology, Fourth Military Medical University, Xi'an, China.
}

Received 30 September 2011; revised 10 November 2011; accepted 27 November 2011.

\section{ABSTRACT}

This study aimed to clarify the ability of the buffer systems of saliva to inhibit enamel demineralization after intake of an acid beverage. In the first experiment, titrable acidity tests were carried out. Ten milliliters of saliva stimulated by chewing gum base was obtained from 10 healthy adult subjects and the $\mathrm{pH}$ of each saliva sample was measured. The beverages used for the experiment were a carbonated soft drink (pH 2.2), a sports drink (pH 3.5), and $100 \%$ orange juice (pH 3.8). Distilled water adjusted to the $\mathrm{pH}$ of each saliva sample was used as a control. In the second experiment, the suppressive ability of saliva against enamel demineralization was quantitatively analyzed using quantitative lightinduced fluorescence (QLF). Aliquots of stimulated saliva obtained from a subject were mixed with $15 \mathrm{ml}$ of $100 \%$ orange juice in saliva:orange juice ratios of $1 / 30,1 / 15,1 / 10$ and $1 / 5$, and bovine teeth were soaked for 24 hours in the solutions. The $\Delta \mathbf{Q}$ of the QLF analyses of the enamel was then measured. The lowest titrant volume which reduced the $\mathrm{pH}$ of the initial saliva (7.7 on average) to $\mathrm{pH} 5.4$ was that of the orange juice. No relationship was found between the buffer capacity and the $\mathrm{pH}$ of the acid beverages. From the QLF measurement, the saliva-orange juice group showed a significantly decreased amount of enamel demineralization ( $p<0.01$ at $20 \%$ level) compared with the distilled water-orange juice group. In conclusion, saliva acts as a buffer to suppress enamel demineralization caused by low-pH beverages.

Keywords: Erosion; Acid Beverage; Saliva; Buffering Capacity; QLF

\section{INTRODUCTION}

A large number of the soft drinks we consume regularly, such as sports drinks, carbonated soft drinks and fruit juices, are acidic ( $\mathrm{pH} 2.2$ or more) and have been shown to cause acid erosion, depending on the amount and pattern of consumption [1,2]. In the human oral cavity, the salivation rate changes substantially, depending on the properties of the ingested solutions [3,4], and the repeated occurrence of intake of a soft drink and salivation to dilute it maintains the homeostasis of the oral $\mathrm{pH}$ environment $[5,6]$.

With regard to the acid buffering capacity of saliva, Lilienthal [7] demonstrated a high acid buffering activity of salivary bicarbonates by titrating hydrochloric acid solution into saliva. Several studies have investigated the acid buffering capacity of saliva [8-10]; however, the acid buffering activity of saliva against low-pH soft drinks and its effect on dental demineralization have not yet been fully elucidated.

In this study, with the aim of obtaining evidence for oral hygiene instruction in dental practice, we performed titration of different types of soft drinks into stimulated saliva and the quantitative observation of soft-drinkinduced demineralization of bovine tooth enamel and the inhibitory effect of saliva on demineralization, using quantitative light-induced fluorescence (QLF) $[11,12]$.

\section{MATERIAL AND METHODS}

This study was performed in accordance with the guidelines of the ethics committee of Meikai University School of Dentistry (approval number: A0913).

\subsection{Experiment 1. Titration of Different Types of Soft Drinks into Stimulated Saliva}

This experiment involved 10 healthy adult subjects, 
consisting of 4 males aged 25 to 40 years and 6 females aged 25 to 40 years, with no tooth defects and who were not receiving any medication. Mechanically-stimulated saliva samples were collected from each subject by instructing them to chew gum base $(1.0 \mathrm{~g})$ and the saliva was immediately subjected to $\mathrm{pH}$ measurement. Ten milliliters each of the collected saliva samples were titrated with Coca Cola (Coca-Cola (Japan) Co., Limited, Tokyo, Japan; $\mathrm{pH}$ 2.2; hereinafter referred to as "the carbonated soft drink"), Pocari Sweat (Otsuka Pharmaceutical, Co., Ltd., Tokyo, Japan; pH 3.5; hereinafter referred to as "the sports drink") and $100 \%$ orange juice (Kirin Tropicana, Co., Ltd., Tokyo, Japan; pH 3.8). Distilled water adjusted with sodium hydroxide to the $\mathrm{pH}$ of each stimulated saliva sample was used as a control. The volume of each titrant required to lower the $\mathrm{pH}$ of saliva and control solutions to 5.4, the approximate critical $\mathrm{pH}$ level for demineralization, was determined and compared among the three types of beverage and between saliva and control.

All saliva samples were collected at 3:00 pm and immediately subjected to $\mathrm{pH}$ measurement. In addition, subjects were instructed to refrain from drinking, eating or smoking for 2 hours before the start of each experiment.

\subsection{Experiment 2. Quantitative Observation of the Demineralization Inhibitory Effect of Saliva on Bovine Tooth Enamel}

Ten bovine maxillary incisor teeth were used in this experiment. A 1-mm square window was formed over one-third of the labial and incisal surface of each tooth with nail varnish. The beverage used in this experiment was $100 \%$ orange juice. A mechanically-stimulated saliva sample was collected from a single subject by instructing him or her to chew gum base $(1.0 \mathrm{~g})$ and the saliva was immediately subjected to $\mathrm{pH}$ measurement. The saliva sample was added to $100 \%$ orange juice to prepare solutions containing saliva at ratios of 1:30, 1:15,
1:10 and 1:5. The sample teeth were soaked in $15 \mathrm{ml}$ each of the saliva-containing orange juice solutions at room temperature for 24 hours while shaking in a water bath shaker (Taitec, Aichi, Japan). Distilled water adjusted to the $\mathrm{pH}$ of the stimulated saliva sample (with sodium hydroxide) mixed with $100 \%$ orange juice was used as control.

Each soaked tooth was washed, dried and subjected to evaluation of the degree of demineralization with a QLF apparatus (Inspektor Research Systems BV, Amsterdam, Netherlands). More specifically, ultraviolet light at a wavelength of $370 \pm 80 \mathrm{~nm}$ was irradiated to the surface of a tooth with visible light blocked, and the fluorescence light reflected from the area near the enamel-dentin junction was allowed to pass through a 520-nm filter and captured by a CCD camera as image data [11]. QLF results were evaluated using $\Delta \mathrm{Q}\left(\% \mathrm{~mm}^{2}\right)$, a parameter representing the mean amount of demineralization.

For statistical analysis of the results of each experiment, the Student t-test was used for two-group comparison and Scheffe's multiple comparison test was used for multigroup comparison.

\section{RESULTS}

\subsection{Experiment 1}

The mean $\mathrm{pH}$ of mechanically-stimulated saliva samples collected from 10 subjects instructed to chew gum base was $7.70 \pm 0.19$. Ten milliliters of each of the saliva samples was titrated with the carbonated soft drink, sports drink and $100 \%$ orange juice. The results of the titration experiment are shown in Figures 1-3. The volumes of the carbonated soft drink and sports drink required to lower the $\mathrm{pH}$ of the saliva samples to the critical $\mathrm{pH}$ level for demineralization were about 7 and 4 times higher than those required to lower the $\mathrm{pH}$ of distilled water adjusted to the same $\mathrm{pH}$ levels, with significant differences between saliva and control $(p<0.01)$ (Figure 4). The volume of $100 \%$ orange juice required

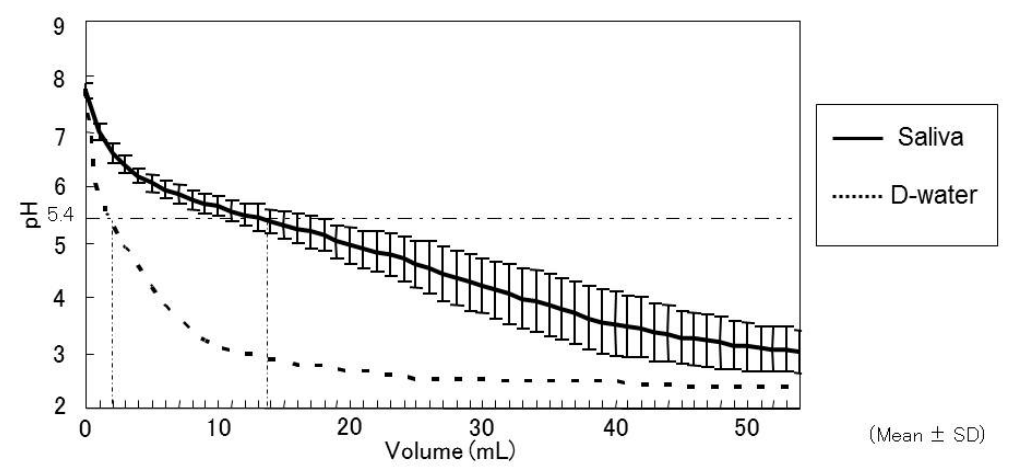

Figure 1. Titration curve with the carbonated soft drink ( $\mathrm{pH} 2.2)$. 


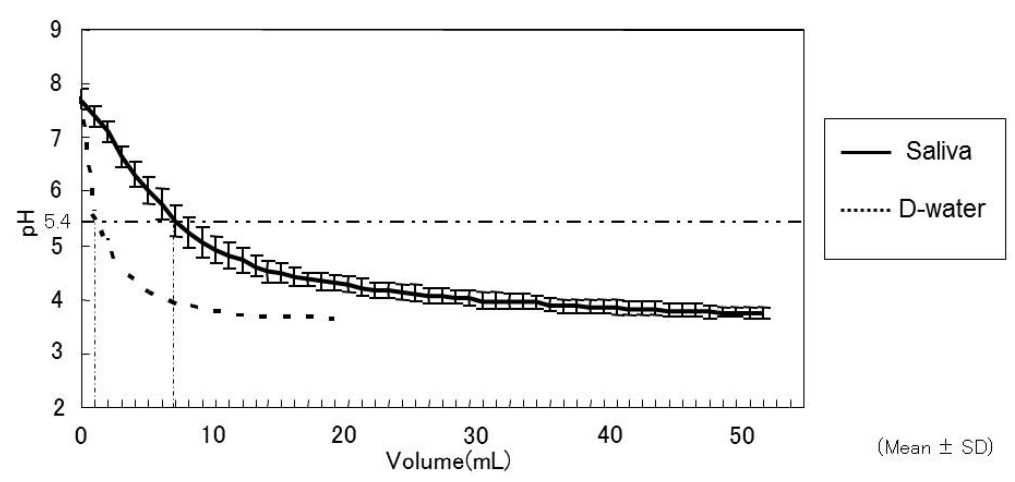

Figure 2. Titration curve with the sports drink ( $\mathrm{pH} 3.5)$.

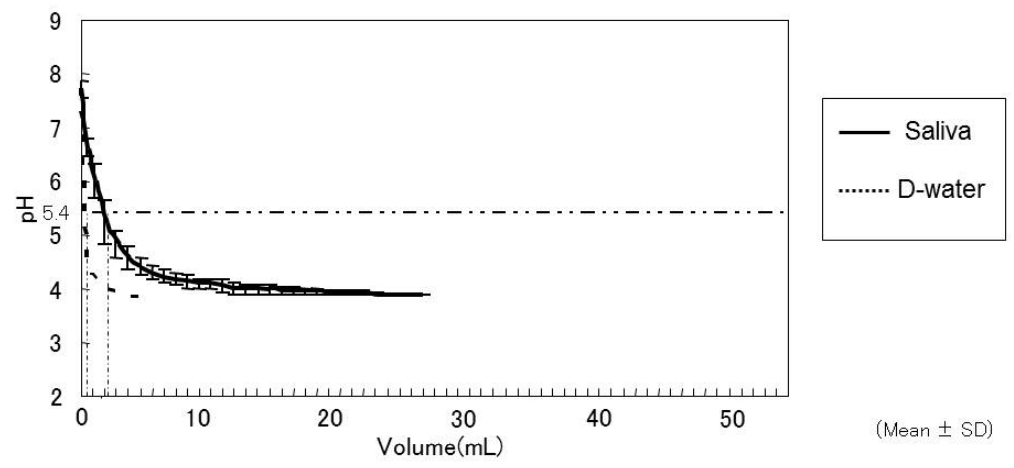

Figure 3. Titration curve with the $100 \%$ orange juice (pH 3.8).

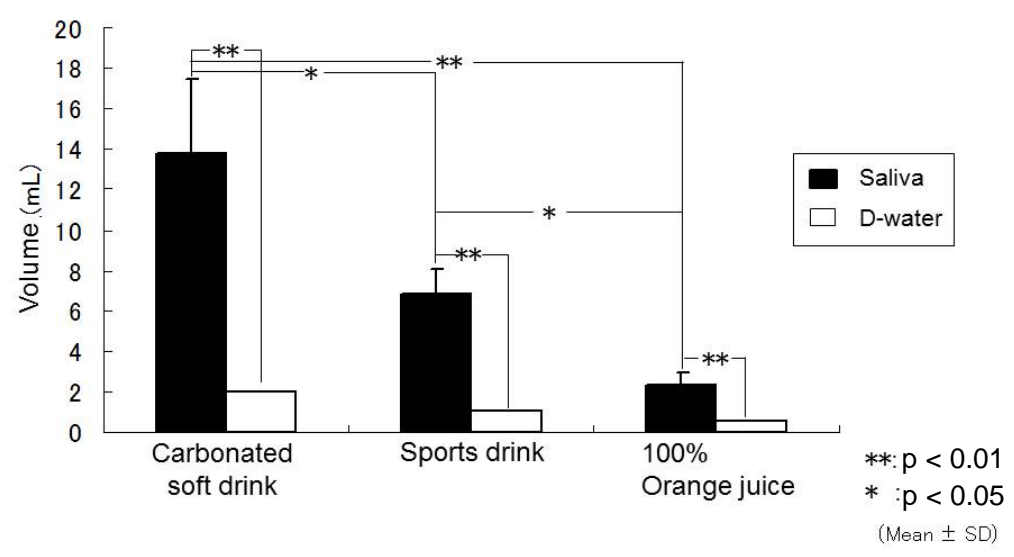

Figure 4. The comparison of volume required to reduce the $\mathrm{pH}$ of saliva to the critical $\mathrm{pH}$ level for demineralization.

to reduce the $\mathrm{pH}$ of saliva to the critical $\mathrm{pH}$ level for demineralization was significantly lower than those of the sports drink and carbonated soft drink.

\subsection{Experiment 2}

The results of a comparison of the degrees of demineralization of sample teeth soaked in $100 \%$ orange juice $(\Delta \mathrm{Q})$, orange juice containing saliva at different ratios $(\Delta \mathrm{Qs})$ and control $(\Delta \mathrm{Qw})$ are shown in Figure 5. Compared with the orange juice containing no saliva, $\Delta \mathrm{Qs}$ values significantly increased and the amount of demineralization significantly decreased with increasing mixture ratio. Although a slight increase in $\Delta \mathrm{Qw}$ was observed, the degree of decrease in demineralization with increasing mixture ratio was lower in saliva-containing distilled water than in saliva-containing orange juice.

A comparison between $\Delta \mathrm{Qs}$ and $\Delta \mathrm{Qw}$ showed that the amount of demineralization in saliva-containing orange juice was lower than that in saliva-containing distilled water at all mixture ratios. A significant difference was 


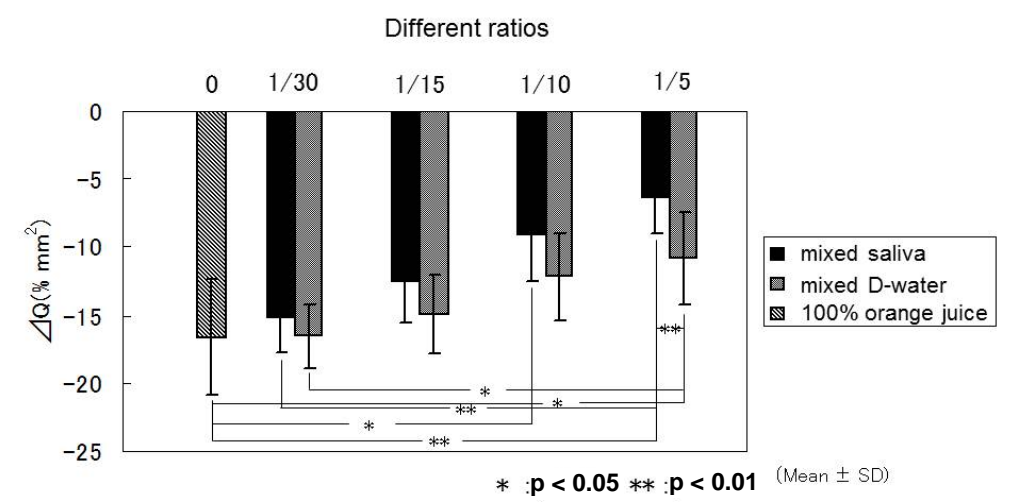

Figure 5. The comparison of the degrees of demineralization of sample teeth soaked in $100 \%$ orange juice (ratio 0 as control), orange juice containing saliva at different ratios.

found between the two solutions at a mixture ratio of $1: 5$ $(p<0.01)$.

The experiment also revealed that demineralization of bovine tooth enamel began after 3 hours of soaking in $100 \%$ orange juice (Table $\mathbf{1}$ ).

\section{DISCUSSION}

\subsection{Acid Buffering Effect of Saliva and Titratable Acidity of Soft Drinks}

In experiment 1, stimulated saliva samples collected from subjects were titrated with three types of soft drinks and changes in the $\mathrm{pH}$ of the saliva samples were measured. The volume of each beverage required to reduce the $\mathrm{pH}$ of distilled water to the critical level of 5.4 was significantly lower than that required to lower the $\mathrm{pH}$ of the saliva samples, clearly demonstrating an acid buffering effect of saliva. The magnitude of the acid buffering capacity of saliva is in proportion to the content of bicarbonate in saliva, which is known to increase with increasing rate of salivation in response to stimuli $[9,13]$. Thus, the mechanically-stimulated saliva samples collected from the subjects instructed to chew gum base are considered to have a higher acid buffering capacity

Table 1. The progression of $\Delta \mathrm{Q}$ of the teeth soaking in $100 \%$ orange juice.

\begin{tabular}{cc}
\hline Soaking time (hour) & $\Delta \mathrm{Q}\left(\% \mathrm{~mm}^{2}\right)$ \\
\hline 0 & 0 \\
1 & 0 \\
3 & $-0.24 \pm 0.13$ \\
4.5 & $-0.39 \pm 0.19$ \\
5 & $-1.54 \pm 0.45$ \\
6 & $-3.70 \pm 1.16$ \\
12 & $-6.47 \pm 2.06$ \\
24 & $-16.61 \pm 4.29$ \\
\hline
\end{tabular}

than unstimulated saliva. Of the three types of soft drinks, $100 \%$ orange juice, which had the highest $\mathrm{pH}$, lowered the $\mathrm{pH}$ of the saliva samples at the earliest time point. The major acid components of each beverage are as follows: phosphoric acid for carbonated soft drinks, citric acid and fruit-derived organic acid for sports drinks, and several types of fruit-derived organic acid for $100 \%$ orange juice drinks. The titratable acidities (buffering capacity) of different fruit juice drinks, including orange juice drinks, which show relatively higher $\mathrm{pH}$ levels than other soft drinks, have been measured by titrating each beverage with a sodium hydroxide solution [14-18]. The results of these studies suggest that the magnitude of the acid buffering capacity of a solution does not necessarily match its $\mathrm{pH}$ levels. The results of the present experiments were also consistent with previous findings. The reason for this has been known, but Larsen and Nyvad suggested that fruit-derived organic acid binds to calcium contained in a solution to form an organic-calcium complex, which exerts a strong buffering effect [16].

\subsection{Demineralization Inhibitory Effect of a Saliva-Containing Soft Drink}

The present study used the $\Delta \mathrm{Q}$ value for evaluation of demineralization. The $\Delta \mathrm{Q}$ value has been shown to be as reliable as the $\Delta Z$ value, which reflects mineral loss in a caries lesion and is used in microradiography [19]. Several studies have employed the $\Delta \mathrm{Q}$ value as a measure of the degree of demineralization $[11,19]$.

The risk for tooth demineralization caused by soft drinks has traditionally been evaluated based only on their $\mathrm{pH}$ levels. The results of the present study suggest the need for reconsidering this risk.

In experiment 2, the amount of demineralization decreased with increasing mixture ratio at a greater degree in saliva-containing soft drinks than in saliva-containing 
distilled water. This result appeared to depend greatly on the acid buffering capacity of saliva. The mixture of stimulated saliva with $100 \%$ orange juice resulted in a greater degree of increase in $\mathrm{pH}$ with increasing mixture ratio than when saliva was mixed with distilled water. The increase in $\mathrm{pH}$ was about 0.1 at a saliva mixture ratio of 1:5. Larsen et al. [20] showed that the amount of apatite crystal that can be dissolved in $1.5 \mathrm{~L}$ of distilled water was $0.5 \mathrm{~g}$ at $\mathrm{pH} 5,5 \mathrm{~g}$ at $\mathrm{pH} 4$ and $85 \mathrm{~g}$ at $\mathrm{pH} 3$, suggesting that at a $\mathrm{pH}$ level of 4 or less, a slight change in $\mathrm{pH}$ can significantly affect the amount of enamel demineralization. The results of the present experiments thus suggest that the acid buffering effect of saliva is a significant factor that inhibits tooth demineralization. The $\mathrm{pH}$ levels of solutions after 24 hours of soaking of bovine teeth were higher by about 2.0 than those immediately after soaking. This may be explained by the previous finding that carbonates are released from the enamel during the process of demineralization and converted into carbonic acid, which increases $\mathrm{pH}$ [20].

The inhibition of demineralization of bovine teeth by the addition of saliva appears to be mediated not only by the aforementioned acid buffering effect of saliva but also by remineralization via the supply of saliva-derived minerals to the demineralized portion. It is known that saliva is supersaturated with respect to minerals, such as hydroxyapatite or tooth enamel and thus the calcium and phosphate ions in saliva serve as remineralization promoting factors. The concentrations of these ions, as well as bicarbonates, are higher in stimulated saliva than in unstimulated saliva. It is thus possible that in the present experiments, minerals contained in the stimulated saliva samples were supplied to the demineralized portion of the bovine tooth enamel, which might have resulted in the observed inhibition of demineralization. Another possible factor for demineralization inhibition is the protective effect of a pellicle formed on the surface of the enamel. The time required for pellicle formation varies substantially depending on the experimental environment. Meurman et al. [21] reported that it takes 7 days until a pellicle is formed in vitro while Hannig et al. [22] reported that it only takes 3 minutes before a pellicle is formed and exerts its protective effect in vivo. In the oral cavity with normal salivation, the tooth enamel is always protected by a pellicle and exposed to saliva supersaturated with respect to tooth minerals. It is thus likely that the demineralization inhibitory effect of saliva is constantly exerted in the oral cavity.

In conclusion, saliva acts as a buffer to suppress enamel demineralization caused by low-pH beverages.

\section{ACKNOWLEDGEMENTS}

The author thanks the subjects for their cooperation and prof. C.
Dawes, Department of Oral Biology, University of Manitoba, Winnipeg, Canada, for helpful suggestions and comments.

\section{REFERENCES}

[1] ten Cate, J.M. and Imfeld, T. (1996) Dental erosion, summary. European Journal of Oral Sciences, 104, 241-244. doi:10.1111/j.1600-0722.1996.tb00073.x

[2] Vissink, A., Burlage, F.R., Spijkervet, F.K., Jansma, J. and Coppes, R.P. (2003) Prevention and treatment of the consequences of head and neck radiotherapy. Critical Reviews in Oral Biology \& Medicine, 14, 213-225. doi: $10.1177 / 154411130301400306$

[3] Watanabe, S. and Dawes, C. (1988) The effects of different foods and concentrations of citric acid on the flow rate of whole saliva in man. Archives of Oral Biology, 33, 1-5. doi:10.1016/0003-9969(88)90089-1

[4] Watanabe, S. and Dawes, C. (1988) A comparison of the effects of tasting and chewing foods on the flow rate of whole saliva in man. Archives of Oral Biology, 33, 761-764. doi:10.1016/0003-9969(88)90010-6

[5] Dawes, C. (1983) A mathematical model of salivary clearance of sugar from the oral cavity. Caries Research, 17, 321-334. doi:10.1159/000260684

[6] Dawes, C., Watanabe, S., Biglow-Lecomte, P. and Dibdin, G.H. (1989) Estimation of the velocity of the salivary film at some different locations in the mouth. Journal of Dental Research, 68, 1479-1482. doi:10.1177/00220345890680110201

[7] Lilienthal, B. (1955) An analysis of the buffer systems in saliva. Journal of Dental Research, 34, 516-530. doi:10.1177/00220345550340040701

[8] Larsen, M.J., Jensen, A.F., Madsen, D.M. and Pearce, E.I.F. (1999) Individual variations of $\mathrm{pH}$, buffer capacity, and concentrations of calcium and phosphate in unstimulated whole saliva. Archives of Oral Biology, 44, 111-117. doi:10.1016/S0003-9969(98)00108-3

[9] Bardow, A., Moe, D., Nyvad, B. and Nauntofte, B. (2000) The buffer capacity and buffer systems of human whole saliva measured without loss of $\mathrm{CO}_{2}$. Archives of Oral Biology, 45, 1-12. doi:10.1016/S0003-9969(99)00119-3

[10] Moritsuka, M., Kitasako, Y., Burrow, M.F., Ikeda, M. and Tagami, J. (2006) The $\mathrm{pH}$ change after $\mathrm{HCl}$ titration into resting and stimulated saliva for a buffering capacity test. Australian Dental Journal, 51, 170-174. doi:10.1111/j.1834-7819.2006.tb00422.x

[11] Ando, M., Hall, A.F., Eckert, G.J., Schemehorn, B.R., Analoui, M. and Stookey, G.K. (1997) Relative ability of laser fluorescence techniques to quantitate early mineral loss in vitro. Caries Research, 31, 125-131. doi:10.1159/000262387

[12] de Josselin de Jong, E., Sundstrom, F., Westerling, H., Tranaeus, S., ten Bosch, J.J. and Angmar-Mansson, B. (1995) A new method for in vivo quantification of changes in initial enamel caries with laser fluorescence. Caries Research, 29, 2-7. doi:10.1159/000262032

[13] Edgar, W.M. (1992) Saliva: Its secretion, composition and functions. Brazilian Dental Journal, 172, 305-312. doi:10.1038/sj.bdj.4807861

[14] Owens, B.M. (2007) The potential effects of $\mathrm{pH}$ and buffering capacity on dental erosion. General Dentistry, 
55, 527-531.

[15] Edwards, M., Creanor, S.L., Foye, R.H. and Gilmour, W.H. (1999) Buffering capacities of soft drinks: the potential influence on dental erosion. Journal of Oral Rehabilitation, 26, 923-927.

doi:10.1046/j.1365-2842.1999.00494.x

[16] Larsen, M.J. and Nyvad, B. (1999) Enamel erosion by some soft drinks and orange juice relative to their $\mathrm{pH}$, buffering effect and contents of calcium phosphate. Caries Research, 33, 81-87. doi:10.1159/000016499

[17] Zero, D.T. and Lussi, A. (2005) Erosion-chemical and biological factors of importance to the dental practitioner. International Dental Journal, 55, 285-290.

[18] Jensdottir, T., Holbrook, P., Nauntofte, B., Buchwald, C. and Bardow, A. (2006) Immediate erosive potential of cola drinks and orange juice. Journal of Dental Research, 85, 226-230. doi:10.1177/154405910608500304

[19] Pretty, I.A., Edgar, W.M. and Higham, S.M. (2002) De- tection of in vitro demineralization of primary teeth using quantitative light-induced fluorescence(QLF). International Journal of Paediatric Dentistry, 12, 158-167. doi:10.1046/j.1365-263X.2002.00357.x

[20] Larsen, M.J., Pearce, E.I.F. and Jensen, S.J. (1993) Notes on the dissolution of human dental enamel in dilute acid solutions at high solid/solution ratio. Caries Research, 27, 87-95. doi: $10.1159 / 000261523$

[21] Meurman, J.H. and Frank, R.M. (1991) Scanning electron microscopic study of the effect of salivary pellicle on enamel erosion. Caries Research, 25, 1-6. doi:10.1159/000261335

[22] Hannig, M., Fiebiger, M., Guntzer, M., Dobert, A., Zimehl, R. and Nekrashevych, Y. (2004) Protective effect of the in situ formed short-term salivary pellicle. Archives of Oral Biology, 49, 903-910. doi:10.1016/j.archoralbio.2004.05.008 